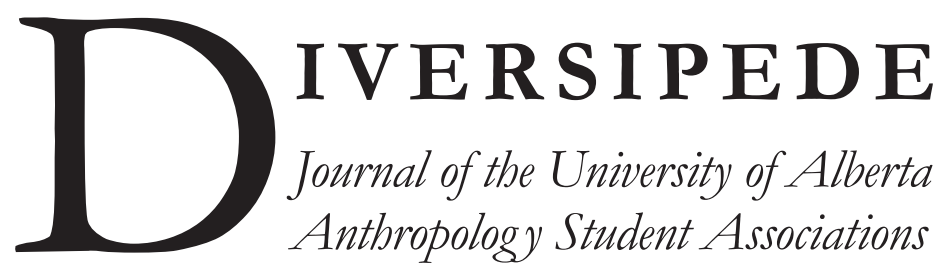

\title{
Touch, Martial Arts, and Embodied Knowledge Scott Habkirk
}

Abstract: Touch is essential to normal buman development. It communicates a wide variety of things depending on the culture and the context. Through touch the boundary between subject and object becomes blurred and empatby can be cultivated. In the context of martial arts, touch can paradoxically lower levels of aggression, particularly in a traditional setting. This paper explores how touch is managed in martial arts and the embodied experiences that it can cultivate. Positivistic methodologies have revealed some of the benefits of martial arts on both physical and mental health, and studies that take a quantitative and culturally sensitive approach are revealing other dimensions of bodily experience.

$\mathrm{T}$ his paper will focus on how the anthropology of the senses, in particular studies about touch, can help us to understand the embodied experience of martial arts. Psychological research done in response to the growing popularity of martial arts in the 1970 s has suggested that martial arts training can increase confidence and assertiveness while lowering levels of aggression. A more phenomenological approach to the study of martial arts has also revealed how culture shapes the practitioners subjective, embodied experiences. As I will outline in this paper, phenomenological methodology, drawing from existing literature and my own experiences, offers another means of exploring experiences of the body through training and fighting. As both an anthropologist and a martial artist, I have experienced firsthand how the anthropology of the senses can help us to understand how social and cultural forces shape the way we experience our bodies.

\section{'Rubbing People the Wrong Way'}

There are many idioms that refer to touch as a form of communication, and researchers who have studied touch in relation to health have observed that touch is essential to normal human development (Andersen 2011; Hertenstein 2011). Touch is necessary for the maintenance of physical and psychological health because it fosters intimacy and emotional connections. It often expresses feelings of warmth and love; in severe cases, touch deprivation can result in emotional, mental, and immunological problems. Touch can also be an act of aggression or coercion, but whether it is attracting or repelling, touch is the medium through which we have some of our deepest sensual experiences. In the edited volume Handbook of Touch (2011), Mathew Hertenstein discusses the crucial role that touch plays in communication. Touch can communicate a wide variety of sentiments that are context-dependent. As a form of communication, touch has both equifinality and equipotentiality. Equifinality re- 
fers to how the same idea can be communicated through different forms of touch. For example, support at a funeral in a typical Euro-Canadian context can be expressed through a handshake or a hug, and is usually coupled with verbal condolences. Equipotentiality refers to how the same touch can be ascribed different meanings that depend on the relationship between the two people touching as well as the situational and cultural context. For example, in Western sports a pat on the bum by a familiar teammate is usually understood as a show of support and encouragement, whereas a pat on the bum by an unfamiliar person in almost any other context can be seen as an unwelcome, aggressive, and highly sexualized gesture. These examples highlight the role touch plays in communicating intimacy. As Hertenstein (2011, 307) notes, "To be perceived positively, the intimacy of the touch must be congruent with the intimacy of the relationship." In this way, touch can indicate the nature of a relationship as well as what stage of intimacy the relationship is at (Finnegan 2005).

The boundary between what is acceptable touch in a specific context between two individuals is carefully mediated by the social norms that govern interpersonal conduct, and in certain circumstances those norms may be suspended (e.g., for functional purposes at a doctor's office). Various cultures differ in their ideas about acceptable levels of public intimacy and touch. Peter Anderson (2011) explored some of the cultural differences in communication through touch and found that latitude, collectivist or individualist cultural orientations, and religion played a major role in determining acceptable levels of intimate touch. He found that cultures that are closer to the Equator tend to touch more often. Andersen attributes this to climatological factors related to the cold that lead to decreased skin sensitivity, less outdoor and social activity, reduced skin availability, and a decrease in sunlight which affects the neuroendocrine system (i.e., lack of sunlight increases probability of depression, aggression, and conflict). In the studies Andersen reviewed, people from northern climates tended to be more serious, uptight, and utilitarian when compared to their southern counterparts.
Andersen also found that East Asian cultures, which tend to me more collectivist rather than individualist, were the least touch oriented. East Asian societies have developed strict rules of conduct, decorum, and decency that often inhibit open displays of affection through touch. Ruth Finnegan $(2005,19)$ notes: "In many Asian countries a mouth-to-mouth kiss in public, with its inevitable physical touching, is considered immoral or disgusting." An obvious comparison is the Japanese bow versus the Western handshake. Both are used for greeting new people, but the Japanese bow involves no touch at all. Lastly, Andersen (2011) looks at the effect that religion has on touch behaviour and found that the more religious a person was, the more likely they were to avoid touch. In many religions, touch is associated with sin and indulgence in profane materiality.

Though these studies help us to understand the social and cultural functions of touch, they primarily use positivistic methods to make broad generalizations that reveal little about the intricate complexity and subjectivity of touch behaviour. Their generalizations may prove useful as a guide, but in the increasing culturally mixed environment that we live in today, the important point to take from these studies is how crucial it is to understand the context through which touch behaviour is made meaningful and how. Even when there are clear normative rules, the rules that govern touch behaviour can change depending on who is involved, the situation, and what function communicative touch is intended to perform. It may be the custom to bow in Japan when meeting business associates, but an internationally educated Japanese businessperson may prefer a handshake with a North American trading partner because of their familiarity with each other and the transmissibility of social norms.

\section{Beyond THE TACTILE}

Research into the anthropology of the senses has gone beyond what is typically considered the realm of touch in sensation. In Haptic Geographies: Ethnography, Haptic Knowledges and Sensuous Dispositions, Mark Paterson (2009) argues that the sense of touch cannot be reduced to tactile sensation alone and develops a broader con- 
ceptualization of touch that includes kinesthesia, proprioception, and the vestibular system. Kinesthesia is the sense of bodily movement that is controlled with the nervous system and includes such functions as muscle tension and relaxation. Proprioception is the spatial framework the body uses to understand where it is relative to other objects as well as where various parts of the body are relative to each other. The vestibular system works with kinesthesia and proprioception to maintain a sense of balance and postural equilibrium, and kinesthesia and the vestibular system work together to manage sensations of inertia and momentum. This haptic system serves to orient the body in space and movement while establishing relationships of subject and object between the body and things external to it. Paterson challenges the classic notions of the senses being bound to particular sense organs as concepts like kinesthesia, proprioception, and the vestibular system use a variety of sensory input to manage bodily awareness. His focus is more on the interrelation of the senses and the continually shifting and culturally variable nature of their construction. He notes that while these senses serve to establish and maintain the relationship between the bodily subject and the objects around it, touch in particular can serve to break down the distinction between subject and object.

Caroline Potter (2008) is another author who explores alternative notions of sense in her participatory research on dancing. Like Paterson, she advocates for an understanding of the senses that goes beyond reduction to specific organ sensory input and claims that the senses are an "intermeshed web of perceptory apparatuses that direct the body's total attention... rather than discreet biological pathways that respond independently to physical stimuli" (Potter 2008, 446). In her discussion of dancer's experiences with the senses, she identifies kinesthesia as moving the body through space and time to achieve a desired end. For the dancers, movement of flow is related to tension and relaxation. Emotional tension can manifest unintentionally in the body to inhibit movement and the body's efficient alignment with gravity.

Both Potter and Paterson advocate for us- ing the body as a research tool in order to understand the embodied experience of the senses. They recognize that language can be limited in expressing the sensations of their research participants and that, in order to truly understand their participants' experiences, anthropologists must immerse themselves in their subjects' sensory world. Like dancing, martial arts are another medium through which anthropologists can explore the relationship between movement, the senses, and culture.

\section{Mind, Matter, and the Martial Arts}

Due to the popularity of Bruce Lee's films and the TV series Kung Fu, interest in martial arts grew considerably in the West in the 1970s. As a result, there was also an upsurge of academic research on martial arts in the 1980s, particularly in the field of psychology. Julian Fuller (1988), in his article "Martial arts and psychological health," which appeared in the British Journal of Medical Psychology, does a particularly good job of summing up the research done on martial arts in the 1980s. He identifies a variety of psychological benefits that martial arts provide including a positive effect on assertiveness and self-confidence, a reduction of juvenile delinquency, and a decrease in aggressiveness. Studies found that there was a negative correlation between skill level and aggressiveness, which Fuller and others attributed to a greater cultivation of empathy through tactile communication. The consistent tactile communication through sparring and the practice of techniques with partners serve to blur the boundaries between self and other. In my own practice of White Crane Kung Fu, instructors encourage us to use touch to extend our senses beyond the confines of our own body and into the body of our opponent to identify weaknesses. These weaknesses make themselves evident through tension and, in order to reduce one's own weakness, we are constantly reminded to relax. As Fuller notes:

Their aim is to teach mental calmness, physical relaxation, absorption of antagonistic force, adaptation to sudden obstacles, timing of effective reaction to 
stressors and one-at-a-time problem solving strategies [1988, 325].

Fuller recognizes, though, that the positive effects of martial arts training are most prominent in traditional settings where schools aim to recognize and reproduce the practices' cultural origins. He criticizes the public image of martial arts as portrayed in popular entertainment in the 1970s and 1980s for misrepresenting them as overly aggressive and instrumental. This portrayal tended to ignore the spiritual and ethical components of martial arts as practiced in their traditional cultural contexts. I would argue that this type of representation has not persisted, and that martial artists have replaced it with an emphasis on the spiritual and ethical components of traditional Chinese martial arts. Producers of movies like the Ip Man series, which was released in 2008, 2010, and 2013, ${ }^{1}$ have endeavored to promote an image of Chinese martial arts as being a holistic health practice that avoids conflict unless it is absolutely necessary. Through these movies, martial artists have engaged with the assumption that Chinese martial arts are about violence and instrumental aggression, and aim to correct what they perceive to be misrepresentations. However, Peter Lorge (2012), in his historical research on martial arts in China, asserts that the concept of Chinese martial arts being solely about selfdefense and self-cultivation is a relatively modern phenomenon. For most of Chinese history, the majority of martial artists were not scholarly monks or non-violent enlightened masters. Martial arts were practiced to satisfy practical military needs among warring states or by the ruling imperial government to maintain power through the threat of violence. From a historical perspective, to portray Chinese martial arts as being devoted to self-cultivation can be as equally distorting as portraying it as purely instrumental violence.

Fuller recognizes the limits of his and his colleagues' positivistic approach to the study of martial arts and suggests there is a need for more culturally sensitive methodologies to be used in researching the effects of martial arts training on health. As well, he points out that more research needs to be done in a non-Western setting for the purposes of comparison. As an addendum to Fuller's review, Peter Columbus (1991) suggests that researchers use a phenomenological approach that focuses on the structures of subjective experience and embodied knowledge rather than discursive. Like Fuller, Columbus recognizes that changing the cultural context of the practice changes the training methods and therapeutic effects. Columbus goes so far as to claim that martial arts originate in an Oriental context (1991), but Lorge (2012) argues that this is not the case; most societies at some point in their history have had a martial art. The Western martial tradition goes back to combat sports such as boxing, wrestling, and pankration at the Ancient Greek Olympic Games (Crane 2004), includes knightly martial orders from medieval times, and is, in modern times, found in aggressive sports like hockey, rugby, lacrosse, and the Ultimate Fighting Championship (UFC)'s mixed martial arts. It may be the case that martial arts became more interwoven with other cultural traditions or became more institutionalized in some societies. Columbus's point becomes increasingly valid when considering that to truly understand a specific martial tradition, a researcher must investigate the tradition as practiced in its original cultural setting as well as its various modern transformations. In recent times, even this approach becomes troublesome because of the free flow of cultural influences and information between societies; this has never been as open and easy as it is today. One may go to China to study kung fu or Japan to study karate and find that schools in the country of origin have been affected by the mixed martial arts tradition of North America. Columbus (1991) does recognize how problematic distinguishing a traditional martial art from a non-traditional one can be and tries to develop some criteria for identification (e.g., national origin acknowledged, spiritual component, enhanced artistry).

In more contemporary research on the Indian martial art kalarippayattu, Ian McDonald (2007) identifies three different ways that the mar-

\footnotetext{
${ }^{1}$ Ip Man was Bruce Lee's martial arts teacher in Hong Kong.
} 
tial art is practiced: as performance art, competitive sport, and bodily practice. As a performance art, kalarippayattu is practiced with an emphasis on the spectacular and often for the purpose of attracting tourists. In this way, kalarippayattu engages in the construction of national narrative building and cultural identification. Lorge (2012, 5) also addresses the connection between martial arts and performance art by noting that "it is because effective violence can be physically elegant and aesthetically pleasing that is has taken on such a broad and large standing place in theatre and film." McDonald points out that this type of martial arts practice may promote the martial art to cultural outsiders, but the lack of practical utility undermines kalarippayattu's legitimacy as a credible martial art. My own experience in Taiwan is similar to McDonald's in this respect. Under the teacher I trained with in Taiwan, we only practiced art forms, and there was never any sparring or practical application employed. The more a martial art is practiced for solely performance purposes, the more likely it is to lose its practical utility.

Practitioners of kalarippayattu as a competitive sport focus primarily on practical applications that fit the rules of global sport (McDonald 2007). In opposition to kalarippayattu as practiced for performance art, this transformation to competitive sport uses instrumental rationality to strip away the cultural context. This methodology is also employed by many schools in North America to serve the needs of the increasingly popular mixed martial arts world as embodied by the UFC. Here, instrumental rationality governs practice, and anything that does not serve those ends is seen a superfluous.

Bodily practice is what McDonald (2007) equates with the more traditional versions of $\mathrm{ka}$ larippayattu and is more holistic in nature. According to McDonald, schools that practice the traditional martial art are similar to temples in appearance, are integrated with Ayurvedic medicine, and combine practical utility with graceful art and ritual. Lorge (2012) argues that the notion of traditional authenticity and claims of any particular style, teacher, or school being more authentic or traditional is mostly a matter of marketing. He also asserts that even the notion of a martial arts style is relatively new in Chinese history, and that martial artists previously used lineage rather than style as a marker of identification. Some modern schools try to trace their lineage to distant historical periods and masters in order to gain legitimacy as a more traditional, authentic martial art. Other schools claim authenticity is demonstrated through success in the ring, while their detractors argue that the rules of competitive sport are so limiting that they cannot accurately demonstrate a school's or style's authenticity. For Lorge, the value of a martial art can only be accurately measured on the level of the individual. If the martial art helps the individual reach the particular goals that they are after in practicing it, be it success in competitive sport, better health, or attracting audiences for performance, then the practice has value.

A traditional martial art is one that adheres to certain key principles that resonate with other cultural elements (be they religious, philosophical, or otherwise) in the specific society from which they originate. The key principles in a traditional practice remain conservative and change little over time while also being flexible enough to remain useful regardless of the historical period. A tradition that persists is one that is both immovable and adaptable, and as Lorge (2012, 4) notes: "Martial arts as a living tradition is like any craft tradition in that skills must be taught, learned, and performed by individuals who innovate while reproducing the tradition."

Both Columbus and Fuller are correct in their assertion that cross-cultural studies which compare martial arts traditions in their cultures of origin are needed to identify the unique therapeutic traits of a particular practice. A researcher or practitioner cannot truly understand the uniqueness of their practice until they have something to compare it to. The passing on of oral tradition has always been a strong component of my kung fu training, and one particular story illustrates this assertion clearly. It is said that a kung fu master and a karate master stood on the edge of a cliff while attackers came at them. The karate master executed a beautiful technique and repelled his attacker with a powerful blow. 
The kung fu master just stepped aside and let his attacker fall to his death. This story serves to illustrate a key difference in these two martial arts traditions' philosophies toward aggression. Karate is believed to oppose force with greater force whereas kung fu yields to strength and attacks weakness. Having not trained in karate, I cannot confirm that this is true, but the story does demonstrate how comparison is used to highlight particular key principles and differences.

To answer Fuller's conclusion that more culturally sensitive methods need to be employed in exploring the positive social-psychological effects of martial arts training, Columbus suggests taking a phenomenological approach and identifies similarities between phenomenological research methods and Zen Buddhism. These similarities include an emphasis on immediate experience and a transcendence of the dualism that is inherent in Western philosophy; it was not until more than a decade later that researchers began to put this suggestion into action.

\section{Martial Arts and Phenomenology}

Researchers who use phenomenology as a methodology advocate for the use of the researcher's body as a tool for understanding embodied experience (Downey 2002; Peterson 2009; Potter 2008; Wacquant 2004). The following are two studies I found that have employed this method to understand martial arts. Greg Downey (2002) studied the Brazilian martial art capoeria, and Loïc Wacquant (2004) conducted participant research in Chicago at a boxing club. Both authors describe the experience as an apprenticeship of the senses that emphasizes learning through doing rather than a reflexive rational understanding. I have also found this to be true in my own experience and am often scolded by my teachers when taking a moment to reflect on the technique I am practicing. In the intensity of a fight or sparring match, there is no time for reflection. Moments of intellectual reflection disengage the mind from the body, and the aim of martial arts training is to make the mind and body act as one. Martial artists practice techniques repetitively to the point of making them reflexive instinct, but as Wacquant $(2004,98)$ notes: "A boxer's ability to cognate and reason in the ring has become a faculty of his undivided organism," and the veteran boxer develops "a cultivated instinct, a socialized animal" (emphasis in original). A novice may react on these cultivated instincts, and martial artists skirting unconsciousness during a fight can rely on this training to get back on their feet, but an experienced martial artist has a measure of control over these instincts and does not act purely by reflex.

According to Wacquant $(2004,71)$, there is "a sort of sensuous intoxication that is key to the education of the apprentice boxer" (emphasis in original). For Downey, this sensing is inherently social and cultural. He is critical of positivistic and objectifying approaches that assume that sensation is pre-cultural and that cultural interpretations occur after primary sensation. Instead, sensation is made distinguishable and meaningful the moment it is perceived. In capoeria, the music is an integral part of the martial art and practitioners must also learn to sing and play instruments. The music sets the tempo of the fight and cues changes in practice and style. A novice typically moves along with the music whereas veterans learn to improvise with the music so as to make their movements less predictable. As in dance, through training and acculturation into capoeria, the body is made responsive to culturally specific sounds and the music is made tactile through kinesthesia.

\section{A WARRIOR's LOVE}

You love your comrade so in war. When you see that your quarrel is just and your blood is fighting well, tears rise to your eye. A great sweet feeling of loyalty and of pity fills your heart on seeing your friend so valiantly exposing his body [Jean de Bueil, 1465, cited in Huizing 1979, 73].

Like many tactile encounters (Finnegan 2005), the beginning of a fight or sparring match is marked by the touching of gloves. This touch serves to ratify the agreement that normally unacceptable levels of violence are now made acceptable, though violence at a martial arts club between sparring partners is strictly governed by 
a sense of reciprocity and equilibrium (Wacquant 2004; Downey 2002). Fighters choose their sparring partners carefully to balance the risk of injury with the potential for growth and the building of bodily capital (i.e., endurance, strength, flexibility, power, and overall health). As Wacquant $(2004,82)$ points out: "The principle of reciprocity that tacitly governs the level of violence in the ring dictates that the stronger boxer not profit from his superiority, but also that the weaker fighter not take undue advantage of his partner's willful restraint." The goal of sparring is to approximate the conditions of a real fight, but fighters and their trainers strive not to spend their fighter's bodily capital needlessly. To unnecessarily risk injury is to waste the bodily capital that the fighter has accumulated through training, but to spar without being challenged does not result in an increase in that capital. Through this process fighters develop an increased tolerance and ability for violence, as well as an increased empathy for others, and sparring partners are made into comrades through the heat of the fight.

There is no better example that demonstrates the role that touch and empathy play (or sensitivity to another person's tension and pain) in martial arts than the exercise called push hands. ${ }^{2}$ Push hands is usually a precursor to sparring and in its most basic form involves two opponents facing each other, shoulders square like a mirror image, and touching each other on the backs of their hands. The opponents are not allowed to move their feet and the goal of the exercise is to push your opponent off balance. In more advanced versions of push hands, opponents may move around and it can evolve into grappling, but for novices the opponents remain stationary. This exercise serves to develop a martial artist's sense of kinesthesia, proprioception, and the vestibular system. Once the exercise starts there is constant movement back and forth, opponents are made acutely aware of how they manage their space because they are not allowed to move their feet, and they must maintain a sense of balance while searching their opponent's body for imbal- ances. In order to reduce tension, practitioners focus on remaining relaxed and using as little strength as possible to redirect attacks. The exercise blurs their sense of self and other through an extending of awareness into their opponent's body to search for tension. Where there is tension, the body relies on strength, and if a practitioner relies on strength, then they quickly become tired. If the body is aligned properly, then antagonistic force travels through the arms and body into the legs and out into the ground, or back to its source-the opponent. Through push hands, martial artists cultivate a greater sensitivity to others' tension and pain while also developing a greater sense of balance, movement, and spatial awareness of themselves.

The increase in empathy that results from martial arts training is not cultivated through tactile contact alone. Training in a martial art is "an ultraindividual sport whose apprenticeship is quintessentially collective" (Waquant 2004, 100; emphasis in original). By entering into the moral community of the gym, a fighter becomes accountable to the trainers and other fighters for their management of bodily capital and the level of violence they bring to the ring. As the fighter builds on relationships developed at the club, the progress of any one fighter becomes a matter of collective responsibility. Fighters often practice techniques together in a group and their movements are synchronized by the trainer running the clock. Like a marching band or a group of dancers, the fighters are made to feel as one body that lifts each person beyond their potential solely as an individual. Through these processes, bonds of brotherhood/sisterhood form and empathetic relationships develop. When you go into the ring with a sparring partner, you want to see them improve, so you enact as much violence as they can take and you hit them where they are weak because when they fight competitively, you do not want them to be susceptible to the same weaknesses. At the same time, you feel guilty if you injure them, and any injury is followed by a reassessment of

\footnotetext{
${ }^{2}$ Push hands is also called sticky hands, though both of these refer to a variety of exercises that involve opponents being in constant contact with each other.
} 
the acceptable level of violence between sparring partners.

Sparring serves the practical ends of preparing for a competitive fight, but also acts as a form of play that has grown from the wrestling and horseplay of boys to a mutual testing of friendly adults who are working together to improve their technique. The ring can serve as a level playing ground where income, class, and status outside the club are made less meaningful, and the hierarchies that develop are based on skill and experience. Martial arts clubs are typically dominated by men, and martial skill is entangled with notions of manliness and power, but women have been welcomed and encouraged at the clubs I have trained at. Lorge (2012) notes that women typically participate less in wrestling and boxing and are more present in East Asian martial arts because these rely less on power and strength. He also comments on the fact that women often play key roles in East Asian martial arts films like Crouching Tiger, Hidden Dragon, so there are more role models for women to aspire to than in Western martial arts. For myself, besides trying to avoid touching genitalia and breasts, there is little difference between how I train with women and men. In the ring, the only thing that matters is martial skill, and to hold back or alter my interaction for any other reason than injury or difference in skill level would disrespect my training partner.

\section{Conclusions}

My love of martial arts comes from the fact that it engages the mind, body, and spirit all in one practice. As psychological research has found, it can have positive effects on mental, emotional, and physical health, though there is a need for cross-cultural comparisons to discover how different cultural contexts affect therapeutic outcomes. One of the main reasons why martial arts have such positive benefits is because they engage practitioners' sense of touch, and through touch, as well as social reinforcement, they cultivate a greater sense of personal awareness and sensitivity toward others. These effects cannot be understood through positivistic methods alone. They must be complemented with qualitative method- ologies to reveal the embodied knowledge and subjective experiences that are cultivated through martial arts and other bodily practices.

\section{References Cited}

Andersen, Peter A. 2011. "Tactile traditions: Cultural differences and similarities in haptic communication." In The Handbook of Touch: Neuroscience, Behavioral, and Health Perspectives, edited by Matthew J. Hertenstein and Sandra J. Weiss, pp. 351-369. New York: Springer.

Columbus, Peter J. 1991. "Psychological research on the martial arts: An addendum to Fuller's review." British Journal of Medical Psychology 64: 127-135.

Crane, Gregory R. (ed.). 2004. Ancient Olympic Events. Perseus Digital Library Project, Tufts University. Electronic resource, http:// www.perseus.tufts.edu/Olympics/sports.html (accessed November 17, 2014).

Downey, Greg. 2002. "Listening to capoeira: Phenomenology, embodiment, and the materiality of music." Ethnomusicology 46(3):487-509.

Finnegan, Ruth. 2005. "Tactile communication." In Book of Touch, edited by Constance Classen, pp. 18-24. New York: Berg.

Fuller, Julian R. 1988. "Martial arts and psychological health." British Journal of Medical Psychology 61:317-328.

Hertenstein, Matthew J. 2011. “The communicative functions of touch in adulthood." In The Handbook of Touch: Neuroscience, Behavioral, and Health Perspectives, edited by Matthew J. Hertenstein and Sandra J. Weiss, pp. 299-327. New York: Springer.

Huizing, J. 1979. The Waning of the Middle Ages, F. Hopman (trans.). Harmondsworth: Penguin.

Lorge, Peter. 2012. Chinese Martial Arts: From Antiquity to the Twenty-First Century. New York: Cambridge University Press.

McDonald, Ian. 2007. "Bodily practice, performance art, competitive sport: A critique of kalarippayattu, the martial art of Kerala." Contributions to Indian Sociology 41(2):143-186.

Paterson, Mark. 2009. "Haptic geographies: ethnography, haptic knowledges and sensuous dispositions." Progress in Human Geography 33(6):766-788. 
PotTer, Caroline. 2008. "Sense of motion, senses of self: Becoming a dancer." Ethnos 73(4):444-465.

WaCQUANT, LoÏc. 2004. Body \& Soul: Notebooks of an Apprentice Boxer. New York: Oxford University Press. 